\title{
Hydrological stability drives both local and regional diversity patterns in rock pool metacommunities
}

\author{
Luc Brendonck, Merlijn Jocqué, Karen Tuytens, Brian V. Timms and Bram Vanschoenwinkel
}

L. Brendonck and K. Tuytens, Laboratory of Aquatic Ecology, Evolution and Conservation, Ch. Deberiotstraat 32, BE-3000 Leuven, Belgium. - M. Jocqué, Rutgers, the State Univ. of New Jersey, 195 University Ave, Newark, NJ 07102, USA, and: Koninklijk Belgisch Inst. voor Natuurwetenschappen (KBIN), Vautierstraat 29, BE-1000 Brussels, Belgium. - B. V. Timms, Australian Museum, Sydney, NSW 2000, Australia. - B. Vanschoenwinkel (buschoen@vub.ac.be), Dept of Biology, Vrije Univ. Brussel (VUB), Pleinlaan 2, BE-1050 Brussels, Belgium.

\begin{abstract}
A main challenge associated with macro ecological gradients such as the latitudinal diversity gradient (LDG) is that proxies of potential underlying processes are often correlated at large scales. One way to reliably identify contributing processes is to show that they can lead to similar responses at local scales. Using a set of invertebrate communities from rock pool clusters along a latitudinal gradient in Australia, we investigated the importance of hydrological stability for explaining both local and regional diversity patterns in this habitat. Results show that, at both local and regional scales, habitat stability in terms of the frequency and length of inundations was strongly correlated to local alpha diversity in individual pools and to gamma diversity at the level of pool clusters. Additionally, partitioning beta diversity into components of nestedness and species turnover revealed that communities in unstable habitats were nested subsets of communities in more stable habitats. Overall, this study provides convincing mechanistic support for the climate stability hypothesis as a potential explanation for the LDG in this system. Results also indicate that when there is enough time for dispersal and colonization, regional processes can be relatively unimportant compared to local processes to explain large scale diversity patterns.
\end{abstract}

Despite more than a century of study, the relative importance of different processes contributing to the latitudinal diversity gradient (LDG) remains the subject of substantial debate (Wallace 1878, Hillebrand 2004, Mittelbach et al. 2007). Besides being attributed to potential artifacts resulting from the shape of the continents (mid domain effects) or the larger available area of the tropics, several theories focus on biotic interactions or historic factors such as variation in speciation rates, the legacy of historical perturbations, the effective time available for evolution (Cardillo et al. 2005) or potential tradeoffs between habitat specialization and dispersal ability (Jocqué et al. 2010). One aspect on which several theories converge is latitudinal variation in energy or climate associated processes. However, as already highlighted by Huston (1999), climatic explanations suffer from several weaknesses. First of all, depending on the conditions and on the intensity of climatic fluctuations, these can both promote and reduce diversity (Mackey and Currie 2001, Fox 2013). Additionally, there are relatively few latitudinal datasets that include complete information on both species diversity and environmental variation. Instead, most studies use rough approximations as proxies for environmental stability such as evapotranspiration or temperature seasonality which, over large spatial scales, typically co-vary with other gradients such as productivity. Consequently, Huston (1999) suggested that, to demonstrate that a certain factor dominantly contributes to the LDG, it must be shown that this factor has similar effects at more localized scales. For instance, although these gradients have been linked to the LDG, neither temperature nor climate stability seem to have important effects on diversity within tropical or temperate regions (Huston 1999). This suggests that the effect of these environmental conditions only appear when gradients are long enough. An additional problem is that, while habitat types are likely to be comparable at local scales, this is often not the case at regional or sub-continental scales which prevents unequivocally attributing diversity variation to a single driving agent. Consequently, there are opportunities for intermediate scale studies that contrast the impact of environmental stability at both small and large spatial scales in similar habitats. Such a design allows to test whether variation in local environmental processes can explain regional diversity patterns such as the LDG (Hawkins and Felizola Diniz-Filho 2004).

In this study, we evaluate the effect of local - within pool cluster - and regional - among cluster - variation in hydrological stability of rock pool habitats on richness and community structure of aquatic macro-invertebrates. Rock pools show strong variation in hydrological stability in terms of the frequency and the length of their inundations. Within pool clusters, this variation is predominantly determined by pool morphometry (Vanschoenwinkel et al. 2009). In turn, at the regional scale, variation in climate can ensure 
that pools with similar basins located on different latitudes differ in hydrological stability. Our study is unique in the sense that the same gradients are present at larger and smaller spatial scales.

This natural model system allows us to test the main hypothesis that the same local process - in this case hydrological stability - may generate diversity patterns at both local (within metacommunity) and regional (among metacommunity) scales, potentially resulting in a latitudinal diversity gradient in this system. We expect that hydroperiods (i.e. lengths of inundations) will be shorter in shallower basins and at higher latitudes characterised by a dryer climate resulting in a gradual loss of species with longer aquatic life cycles (Schneider and Frost 1996). Such a nested pattern along a gradient of hydrological stability has been demonstrated in a rock pool cluster with a set of early successional species gradually being complemented by a set of late successional species with longer aquatic life cycles in more stable long lived pools (Vanschoenwinkel et al. 2013). Here, we expand on these findings by testing whether latitudinal variation in climate can generate a similar nested pattern at a regional scale or, alternatively, a pattern of species turnover. The former pattern implies that species are gradually removed as you move to dryer conditions at northern latitudes while the latter implies that species are replaced. If dispersal is not limiting in this region - i.e. all species had the opportunity to colonize all pool clusters - we expect that local nested community patterns generated by variation in hydrological stability among pools could be mirrored at a regional scale due to latitudinal variation in the hydrological stability among pool clusters. On the other hand, regionalism in species assemblages with different sets of species adapted to different hydrological conditions could result in species turnover along this latitudinal gradient. To verify to what extent species composition changes with hydrological stability, we investigate the topology of species $\mathrm{x}$ site matrices sorted along this gradient and partition beta diversity (i.e. variation in species composition among sites) associated with variation in hydrological stability at local and regional scales in components of turnover and nestedness. This allowed us to examine whether compositional changes were due to a gradual reduction of a nested set of species (species removal) or, alternatively, involved species turnover (species replacement).

\section{Material and methods}

\section{Study system}

The study system consists of aquatic macro-invertebrate communities residing in clusters of temporary rock pools on the summit of granite inselbergs (isolated escarpments of outcropping rock) along a $800 \mathrm{~km}$ sub-continental climate gradient in the winter-rainfall region of Western Australia (Fig. 1). In this region, freshwater ecosystems are almost exclusively restricted to rain-filled depressions that occur on inselbergs that lie scattered in an otherwise flat and open landscape (Withers 2000). Previous research has shown that invertebrate communities within these pool clusters - which can span several hundreds of meters - interact with each other via dispersal at ecological time scales (Vanschoenwinkel et al. 2008, 2011). Consequently, invertebrate assemblages in rock pool clusters can be considered metacommunities (Vanschoenwinkel et al. 2007, 2013). On the other hand, dispersal among pool clusters on different inselbergs is very rare and was shown to be only relevant on evolutionary time
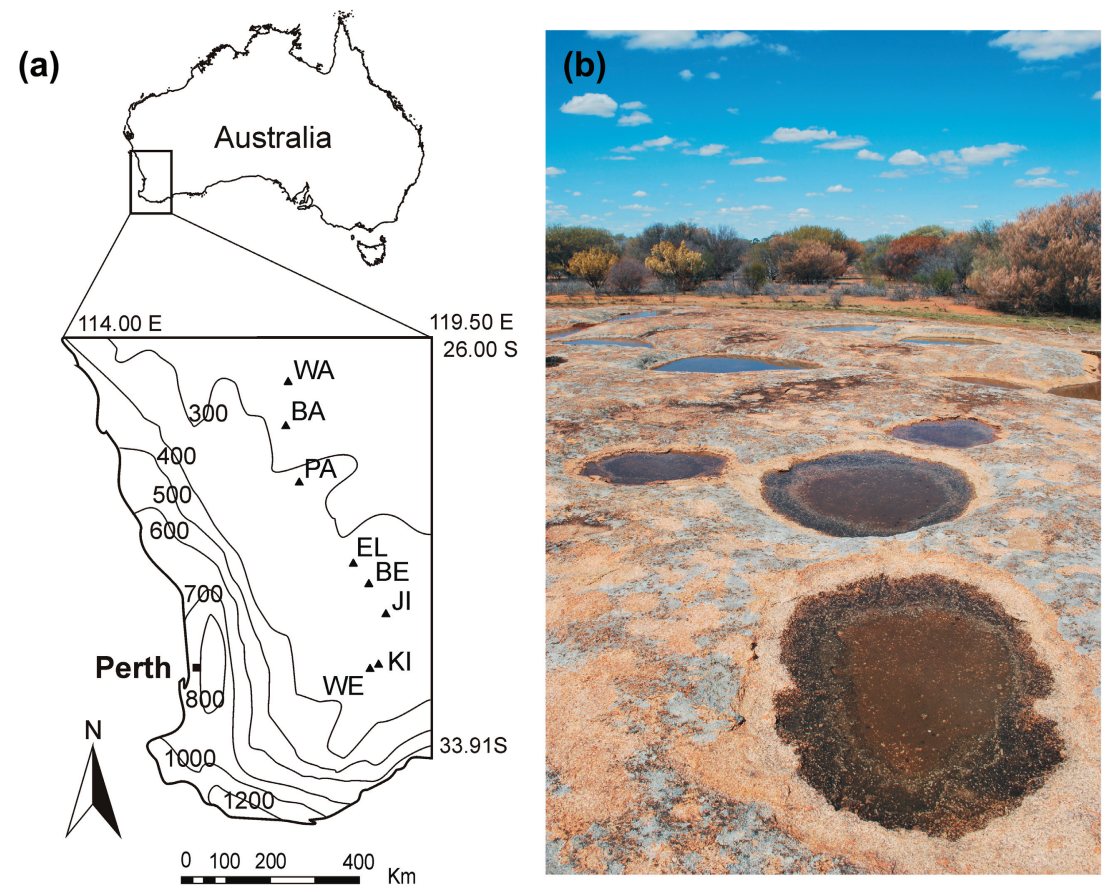

Figure 1. (a) Location of the study area in Western Australia showing the eight inselbergs (triangles) included in this study: Hyden Rock (WE), Kings Rocks (KI), Jilbadgie Rock (JI), Baladgie Rock (BE), Elachbuttin Rock (EL), Bulamanya Rock (PA), Ballan Rock (BA) and Walga Rock (WA). (b) A picture of a typical temporary rock pool cluster at the summit of an inselberg in Western Australia (photo: Bram Vanschoenwinkel). 
scales (Vanschoenwinkel et al. 2011, Pinceel et al. 2013). All inselbergs in the study area are originally derived from the Yilgarn craton: a massive ancient granite body that constitutes the bulk of the land mass of Western Australia. While this granite was formed from intruding magma below the surface 250 m.y.a., inselbergs only emerged much later in the landscape around 50-100 m.y.a. after the granite was fragmented and after the covering sediments were removed by erosion (Twidale and Bourne 1975). Current evidence supports that pool basins already formed in the granite when it was still below the surface due to sub surface weathering (Twidale and Bourne 2001). These depressions were therefore initially filled with soil and covered with vegetation; examples of which are still found in the moist parts of southwestern Australia (Bayly 2011). However, dry conditions such as these present in the inland of Western Australia can result in loss of vegetation and exposure of rock pool basins. Genetic data suggest that the rock pools in the study area were colonized by freshwater invertebrates at least as early as 8-4 m.y.a. (Pinceel et al. 2013). Besides the relatively simple physical structure and clear spatial delineation of both individual communities (rock pools) and metacommunities (rock pool clusters), the presented study system has the advantage that it consists of comparable, geologically identical habitats of similar age occurring over a wide geographic range, minimizing possible confounding effects of other habitat properties that could vary with latitude besides the gradient of interest (hydrological stability). Average annual precipitation decreases with decreasing latitude from Hyden Rock in the south to Walga rock in the north (Fig. 1, Supplementary material Appendix 1 Table A1), while average daily evaporation increases (Supplementary material Appendix 1 Fig. A1, Table A2).

Temporary rock pools depend directly on precipitation for filling and the impermeable eroded basins lose water only via evapotranspiration and to a much lesser extent as a result of drinking animals such as kangaroos. The size (typically correlated with catchment) and depth of pool basins largely determines the volume of water and the potential duration of the inundated phase (Vanschoenwinkel et al. 2009). Since the length of the inundation period (hydroperiod) and the frequency of inundations with a certain hydroperiod are important determinants of community structure in temporary pools (Wiggins et al. 1980, Wellborn et al. 1996, Urban 2004), community composition and richness among communities in a metacommunity may vary considerably, depending on basin depth and associated hydrological stability. Basins with comparable morphometries can, however, experience different filling patterns under different precipitation and evaporation regimes as occur along the studied latitudinal gradient. Consequently, rock pool basins located on inselbergs in the north may have a different inundation pattern than identical basins in the south of the study region (Fig. 1). As such, climate is expected to have a strong impact on the composition of rock pool communities in climatologically different regions.

\section{Sampling design and data collection}

Samples were collected from eight inselbergs each containing a single rock pool cluster, along a $800 \mathrm{~km}$ transect from Hyden Rock near the town of Hyden in the south to Walga
Table 1. (a) Linear regression models explaining variation in taxon richness of metacommunities based on hydrological stability (PC1). (b) multiple regression model explaining variation in local taxon richness based on hydrological stability (PC1) and habitat size (LogArea).

(a) Metacommunity richness

Lin. regression: $F_{1,6}=80.88, p=0.0001, r^{2}=0.92$

\begin{tabular}{lccrr}
\hline & Est. & SE & \multicolumn{1}{c}{$\mathrm{t}$} & \multicolumn{1}{c}{$\mathrm{p}$} \\
\hline Intercept & 24.4 & 0.72 & 34.13 & $<0.0001$ \\
Hyd. stability (avg -PC1) & 3.05 & 0.34 & 8.99 & 0.0001 \\
\hline
\end{tabular}

(b) Local richness

Mult. regression: $F_{2,21}=9.83, p=0.0009, r^{2} a d j=0.43$

\begin{tabular}{lllcc}
\hline & Est. & SE & $t$ & $p$ \\
\hline Intercept & 9.42 & 1.62 & 5.81 & $<0.0001$ \\
Hyd. stability (-PC1) & 0.93 & 0.3 & -3.1 & 0.005 \\
LogArea & 2.47 & 1.25 & 1.98 & 0.06 \\
\hline
\end{tabular}

Rock near the town of Cue in the north (Fig. 1, Table 1). Sampling took place in the winter of 2004 (3-7 August). To assess local - within cluster - variation in hydrological stability three pools were sampled that differ in the depth of their basin (shallow $<6 \mathrm{~cm}$, intermediate $6-15 \mathrm{~cm}$, deep $>15$ $\mathrm{cm})$. Basin depth can be considered a main determinant of local (within pool cluster) variation in stability as deeper basins tend to dry out less frequently and experience a longer hydroperiod (Vanschoenwinkel et al. 2009). Secondly, to quantify diversity at the level of the metacommunity, a pooled community sample was taken from ten representative pools on the inselberg. These 10 pools were selected to capture the range of environmental conditions present on each inselberg (small versus large pools, pools with and without vegetation, centrally located versus more marginal pools). Previous research has shown that sampling ten pools suffices to reach the plateau of a species accumulation curve in rock pool clusters in this region (Jocqué et al. 2007). Aquatic macro-invertebrates were sampled using two different sampling methods. Benthic and pelagic invertebrates were collected by placing a quadrat (surface: $0.5 \mathrm{~m}^{2}$; height: $0.4 \mathrm{~m}$ ) at random locations in each pool. After stirring up the sediment, the equivalent of the enclosed water volume was removed with a measuring cup. The water was subsequently poured over a sieve with a $64-\mu \mathrm{m}$ mesh. The whole sample was preserved in $70 \%$ ethanol. Quadrats were placed once in the shallow, twice in the intermediate and three times in the deep pools. This design was aimed at sampling a similar proportion of habitat in each pool type. However, it still ensured that in small shallow pools almost everything was sampled while a larger proportion of habitat was not sampled in larger, deeper pools. Hence, any positive effects of hydroperiod on diversity are still likely to be underestimated. Macro-invertebrates were sampled with a kick sampler $(500-\mu \mathrm{m}$ netting). Scoops were performed with the same speed covering a distance of about $1 \mathrm{~m}$. We aimed at covering the whole surface of the pool once. Samples were sorted in the field and preserved in $70 \%$ ethanol. For the pooled metacommunity sample of the 10 pools we applied the same protocols. The only difference was that the quadrat was placed only once in each pool and that a fixed number of five kick samples were taken in each pool, regardless of pool size. These samples 
were pooled together. Samples were processed in the laboratory using a stereomicroscope. Due to the relatively limited taxonomic knowledge of the rock pool fauna in this region, reliable identification to species level was often impossible. Therefore we decided to usegenera as the taxonomic units in our analyses. However, for some groups such as harpacticoid copepods, Diptera, Turbellaria and Acari we were restricted to a lower taxonomic resolution at the level of families and orders.

Data collected using the two sampling strategies were combined and merged together in a single presence/absence matrix. Earlier limnological studies of these rock pools confirmed that standard water quality variables such as conductivity and $\mathrm{pH}$ do not show variation along this latitudinal gradient (Timms 2012). Additional data supporting this claim are provided in the electronic supplementary material (Supplementary material Appendix 1 Table A3).

\section{Hydrological stability}

Since rock pools on inselbergs are small (typically $<10 \mathrm{~m}^{2}$ ), impermeable and groundwater independent, it is feasible to reconstruct the hydrological stability of these systems using simple simulation models based on pool morphometry and evaporation and precipitation data from weather stations (Hulsmans et al. 2008, Altermatt et al. 2009). Here, we applied a published hydrological model (Vanschoenwinkel et al. 2009, Tuytens et al. 2014) to obtain both a measure of hydrological stability for individual pools as well as an average measure for each inselberg pool cluster. The model is a stepwise simulation model that predicts daily water levels based on daily rainfall data, and monthly averages of daily evaporation data (Supplementary material Appendix 1 Table A2), basin depth, basin surface area, and two pool specific parameters calculated following the procedure in Tuytens et al. (2014). For each of the sampled pools, basin depth was measured using a calibrated rod. Basin bottoms were generally horizontal. Pool area was estimated by measuring the main axes of the pool basins and using ellipse approximations. Additionally, the same morphometric variables were also measured using the same protocol for 100 extra rock pools on each inselberg. For each inselberg pool cluster, long term climate data were obtained from the nearest weather station. These included daily precipitation $(\mathrm{mm})$ and daily (Class A pan) evaporation (mm) (Bureau of Meteorology; $<$ www.bom.gov.au $>$ ). A list of the weather stations used and their distance to respective inselbergs is provided in Supplementary material Appendix 1 Table A1. Based on 30 years of daily precipitation and evaporation, the model generated a set of hydrological variables for each of the sampled pools as well as for 100 extra pools on each inselberg over this time period including mean, SD, minimum, maximum and median hydroperiod and mean, SD, minimum, maximum and median desiccation frequency (number of times a pool dries out per year). Principal component analysis (PCA) was used to reduce the dimensions of this dataset and define a natural gradient of habitat stability reflected in the dominant first PC axis. Given that the first PC axis reflects the bulk of hydrological variation among pools, site scores of different pools along this axis can reliably be used as a measure of hydrological stability. Here, site scores were multiplied by -1 to ensure that higher scores correspond to more stable sites. To quantify inter metacommunity (i.e. among inselberg) variation in habitat stability along the latitudinal gradient the arithmetic mean of the PC scores of 100 pools of each inselberg along the aforementioned first $\mathrm{PC}$ axis was calculated.

\section{Data analysis}

To confirm a link between pool morphometry, climate and hydrological stability, a general linear model (GLM) was constructed including basin type (shallow, intermediate, deep), latitude and their interaction as predictor variables and hydrological stability (PC1) of each pool as a response variable. Repeating this analysis using a continuous predictor of pool morphometry such as basin depth led to consistent results. However, we decided to use a categorical variable since this matches with the sampling design (one pool of each depth category sampled on each inselberg). Diversity analyses focus on explaining variation in both alpha diversity (i.e. the number of taxa present at a site) and beta diversity (i.e. variation in taxonomic composition among sites). Gamma diversity refers to the total number of taxa found in a region. To test for the effect of hydrological stability on the diversity contained in rock pool metacommunities, the effect of average hydrological stability of each inselberg pool cluster (average PC1 for 100 pools) on the richness of macro-invertebrates sampled from 10 pools in each cluster (metacommunity richness or gamma diversity) was tested using a linear regression model. Additionally, using a similar linear regression model, the effect of hydrological stability (PC1) of individual pools on local richness (alpha diversity) was investigated. To correct for potential confounding effects of habitat size, LOG transformed surface area of each basin was included as an additional predictor variable. Non significant interaction and quadratic terms were removed from final models.

\section{Community composition}

Redundancy analysis (RDA) models were reconstructed to test for effects of average hydrological stability of each pool cluster on the composition of a pooled sample from 10 pools (metacommunity composition). For this, a response matrix of presence-absence data were Hellinger transformed as recommended by Legendre and Gallagher (2001) because they are more representative for actual distances in a multivariate space. To test for potential effects of hydrological stability on local community composition in individual pools, an additional model was built with permutations being restricted within inselbergs using a split plot design (strata command in the rda function) to correct for the fact that certain basins are found on the same inselberg. To assess whether this variation in community composition reflects a nested pattern (with communities from less stable habitats as nested communities from more stable habitats), variation in metacommunity composition captured in the Sørensen dissimilarity index (betaSOR) was partitioned into components that correspond to turnover (betaSIM) and nestedness (betaSNE), respectively, according to the method developed by Baselga (2010) and implemented by Baselga and Orme (2012). This approach allows to assess and 
visualize which fraction of the beta diversity among sites can be attributed to taxon addition and removal (nested patterns) or patterns of turnover (betaSOR $=$ betaSIM + betaSNE). This method was applied, both for variation in composition among metacommunities, as well as for variation in composition of individual rock pool communities. Variation in community composition among metacommunities was visualized using non metric multi dimensional scaling (NMDS) ordination plots based on the same dissimilarity index. Due to high stress levels associated with illustrating variation in two dimensions, NMDS was not suitable to plot variation in composition of individual communities. Consequently, cluster analysis was used instead. Single linkage (nearest neighbor) was used as an amalgamation rule since this is a more effective way to visualize gradients (chains) compared to complete linkage or average agglomerative clustering methods (Borcard et al. 2011).

\section{Nestedness}

Complementary to the partitioning of beta diversity in components of nestedness and turnover, the hypothesis that metacommunities from the north are nested subsets of metacommunities in the south was also assessed using rigorous statistical tests. For this we sorted the taxon $\times$ inselberg matrix based on the average hydrological stability (average PC score for 100 pools in each metacommunity) and tested whether this matrix is significantly nested. Additionally, in order to verify whether hydrological stability is a likely explanation for a nested structure we also tested this prediction at the scale of individual pool communities. For this we sorted a taxon $\times$ pool matrix including all pools in the dataset according to hydrological stability (PC1). To test for a potentially nested structure of these two matrices the NODF nestedness metric was used which is based on overlap and decreasing fill (nestednodf) (Almeida-Neto et al. 2008). Use of other indices such as matrix temperature (Rodríguez-Gironés and Santamaría 2006) and the discrepancy index (Brualdi and Sanderson 1999) led to consistent results. Significance was tested by comparing observed values with those produced by 999 null models generated using the oecosimu function. Presented significances were calculated using a null model that keeps both column and row totals fixed (FF model), recommended by Ulrich and Gotelli (2007) as a conservative algorithm (low type I error) to detect patterns of nestedness.

All analyses were performed in $\mathrm{R}$ ver. 2.14.2 using the packages VEGAN (RDA; redundancy analysis, nestednodf; nestedness analyses), (betapart; partitioning of dissimilarities) and STATS (hclust; hierarchical clustering).

\section{Results}

\section{Latitude, pool basin depth and hydrological stability}

Taking into account differences in evaporation and basin properties of individual pools, hydrological simulations showed that the average hydrological stability of inselberg pool clusters decreases from south to north (Est.: $-1.19 \pm$ $0.04, \mathrm{t}_{(1,6)}=-27.38, \mathrm{p}<0.0001, \mathrm{r}^{2}=0.99$; Supplementary material Appendix 1 Fig. A1). When looking at individual pools, deeper pool basins were generally more stable than shallower ones and pools located in the south of the gradient were more stable than pools in the north (GLM: basin type effect: $F=7.86, p=0.003$; latitude effect: $F=66.77$, $\mathrm{p}<0.0001$; Supplementary material Appendix 1 Fig. A1). There was no significant interaction $(p>0.05)$ illustrating that, regardless of basin type, latitude reduced stability from south to north. The first principal component axis reflected $74 \%$ of total variation in model reconstructed hydrological variables and (when multiplied by -1 ) could be considered as a measure of hydrological stability, with higher scores corresponding to pools that have longer hydroperiods and also experience more inundations per year (Supplementary material Appendix 1 Fig. A2).

\section{Richness and composition of communities and metacommunities}

A total of 57 taxa were recorded (Supplementary material Appendix 1 Table A4, A5). A simple linear regression model showed strong and significant positive effects of average hydrological stability on metacommunity richness. Similar positive effects of the hydrological stability of individual pools were found for local richness (Table 1, Fig. 2). Hydrological stability significantly explained a substantial proportion of variation in community composition of the regional metacommunities on inselbergs (33\%; Table 2a). Variation in local communities on each inselberg was shown to be more related to habitat size (16\%; Table $2 \mathrm{~b})$. The correlation between LOGtransformed surface area and hydrological stability of the pools (captured in PC1) is not significant although there is a trend (Pearson $r=0.33, p=0.11$ ). This explains why both variables can be significant predictors in our diversity analyses.

\section{Nestedness along gradients of hydrological stability}

Both turnover and nestedness patterns contributed to overall variation in the composition of metacommunities and communities. Variation in the taxonomic composition of the eight metacommunities visualized using NMDS reflected variation in average hydrological stability associated with latitude, with higher similarities (shorter distances) between metacommunities situated closer to one another along this gradient (Fig. 3a). The same pattern was preserved in the nested component of this index (Fig. 3b) which accounted for $38 \%$ of beta diversity but not in the turnover component (Fig. 3c) of the index, which accounted for $62 \%$ of beta diversity. Additionally, the ordination of nested component of beta diversity also conserved the latitudinal sequence of inselbergs (from south to north: WE, KI, JI, BE, EL, PF, BA, WA). A similar NMDS representation was not possible for individual pool communities due to very high stress levels associated with plotting dissimilarities in two dimensions (stress $>0.30$ ). Therefore cluster analysis was used instead. Here, the nestedness fraction accounted for $22 \%$ of variation while the turnover fraction represented $77 \%$. Overall, the clustering diagram based on the nested fraction of Sørensen dissimilarity showed a trend of pools with low (lighter symbols) and high hydrological stability (darker symbols) clustering together (Supplementary 

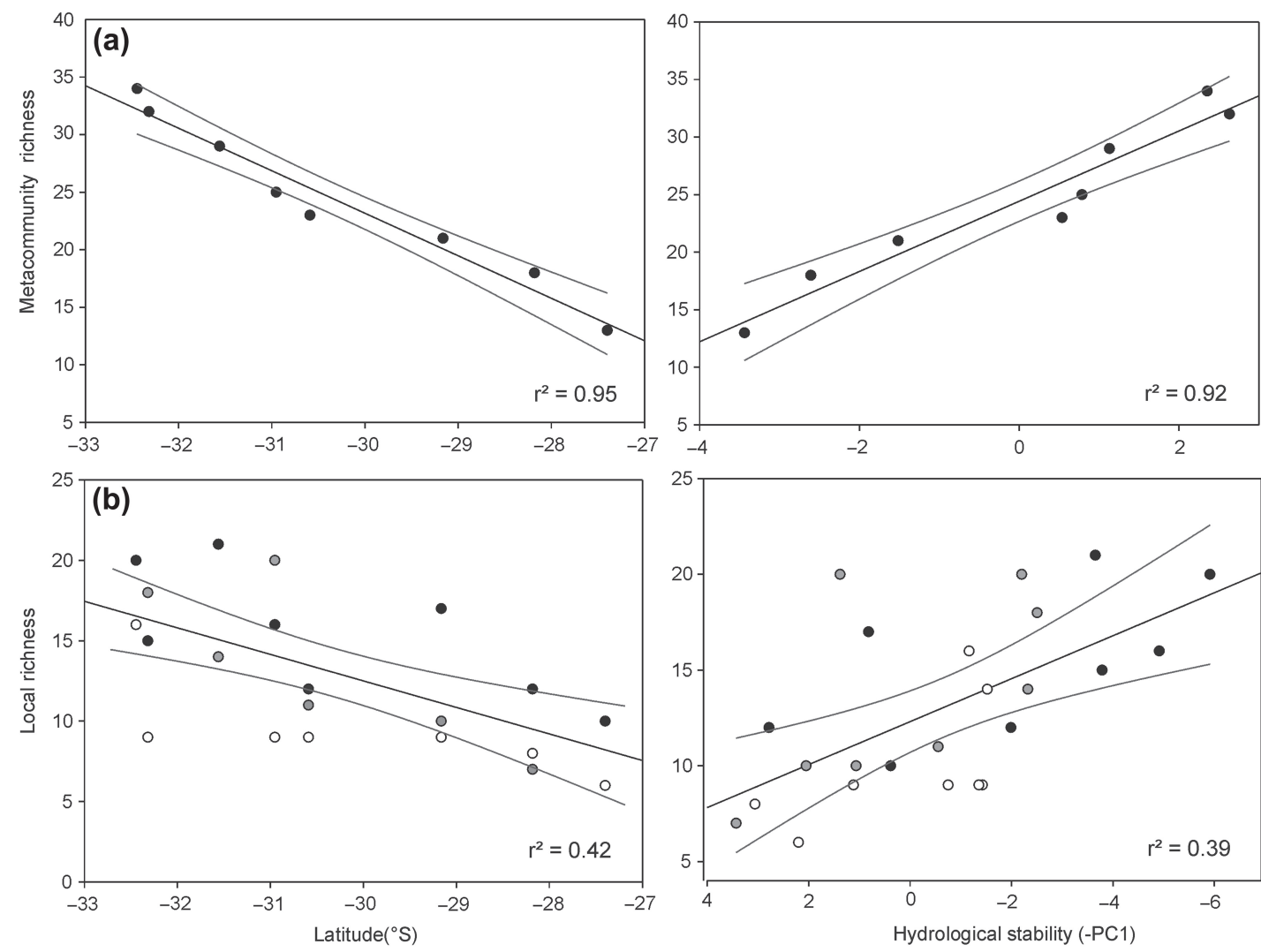

pool type: deep $\bullet$ intermediate $\circ$ shallow o

Figure 2. Scatterplots showing associations between latitude (left panels), hydrological stability (right panels) and richness of freshwater invertebrates present in (a) a pooled sample of 10 communities on eight inselbergs (metacommunity richness) and (b) the invertebrate richness present in three basins (shallow, intermediate, deep) at different latitudes. Regression lines are presented with $95 \%$ confidence intervals. In the bottom panels open circles represent pools with shallow basins $(<6 \mathrm{~cm})$, black filled circles correspond to pools with deep basins $(>15 \mathrm{~cm})$ and grey filled circles designate pools with an intermediate basin depth $(6-15 \mathrm{~cm})$. Hydrological stability of inselbergs (metacommunity level) was calculated as the average first PC score calculated based on model reconstructed hydrological variables for 100 randomly selected pool basins on this inselberg. Coefficients of determination presented in the panels correspond to the fits of the depicted regressions, not the $\mathrm{r}^{2}$ of the multiple regression models in Table 2.

material Appendix 1 Fig. A3). On the other hand, inspection of the clustering diagram reflecting dissimilarities based on turnover revealed some regionalism with, for instance, communities from King Rocks (KI) clustering together. The

Table 2. (a) Redundancy analysis (RDA) models explaining variation in taxonomic composition of metacommunities based on hydrological stability (PC1), respectively. (b) RDA models explaining variation in local community composition found in different types of pool basins (shallow, intermediate, deep) on different latitudes and variation in local community composition based on hydrological stability (PC1) and habitat size (LogArea).

(a) Metacommunity composition

RDA: $F_{1,6}=2.94, p=0.005, r^{2} \mathrm{adj}=0.33$

\begin{tabular}{lcc}
\hline & $F$ & $p$ \\
\hline Hyd. stability (avg -PC1) & 2.94 & 0.005
\end{tabular}

(b) Local community composition

RDA: $F_{3,20}=3.20, p=0.005, r^{2} a d j=0.16$

\begin{tabular}{lcl}
\hline & $F$ & \multicolumn{1}{c}{$\mathrm{P}$} \\
\hline Hyd. stability (-PC1) & 4.93 & 0.32 \\
LogArea & 1.99 & 0.005 \\
\hline
\end{tabular}

association between hydrological stability gradients and nestedness is also supported by analyses of community based and metacommunity based analyses of taxon $X$ site matrices. A taxon $\times$ inselberg matrix containing taxa from each of the eight inselberg metacommunities sorted based on average hydrological stability associated with the latitudinal gradient resulted in a significantly nested matrix with an NODF index of $50.54(\mathrm{z}=4.54, \mathrm{p}=0.005$; Supplementary material Appendix 1 Table A4). Metacommunities in the north with less stable pools were nested in metacommunities in the south that, on average, have more stable pools. Similarly sorting a site $\times$ taxon matrix based on the hydrological stability of each of the 24 individually sampled communities spread over eight inselbergs resulted in a significantly nested structure with an NODF index of $40.24(\mathrm{z}=2.01$, $\mathrm{p}=0.005$; Supplementary material Appendix 1 Table A5) and with communities from less stable pools nested in communities from more stable pools. It must be noted, however, that the relative importance of turnover and nestedness is to some extent confounded by taxonomic resolution. The relative importance of turnover is likely to become larger if taxonomic resolution would have been higher e.g. with the aid of barcoding techniques. 
(a) Sörensen dissimilarity (100\%)

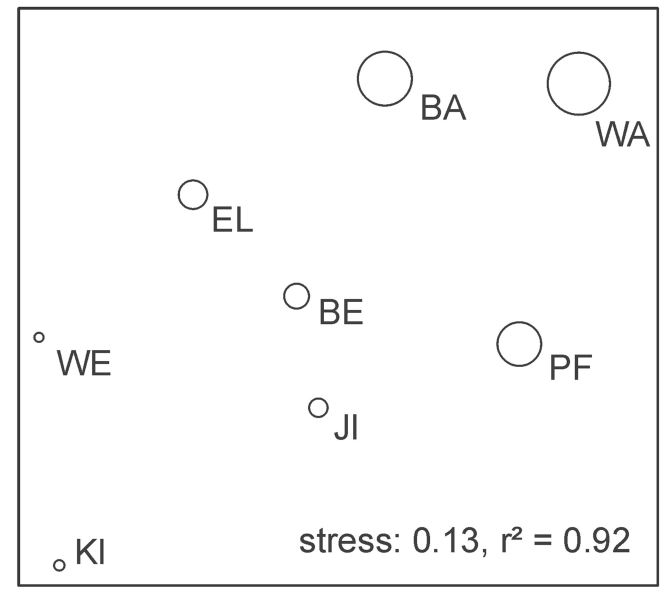

(b) Nestedness fraction (38\%)

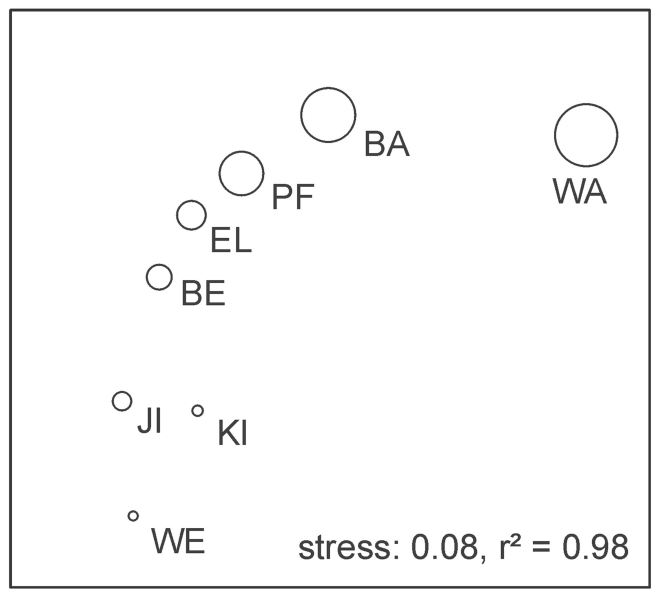

(c) Turnover fraction (62\%)

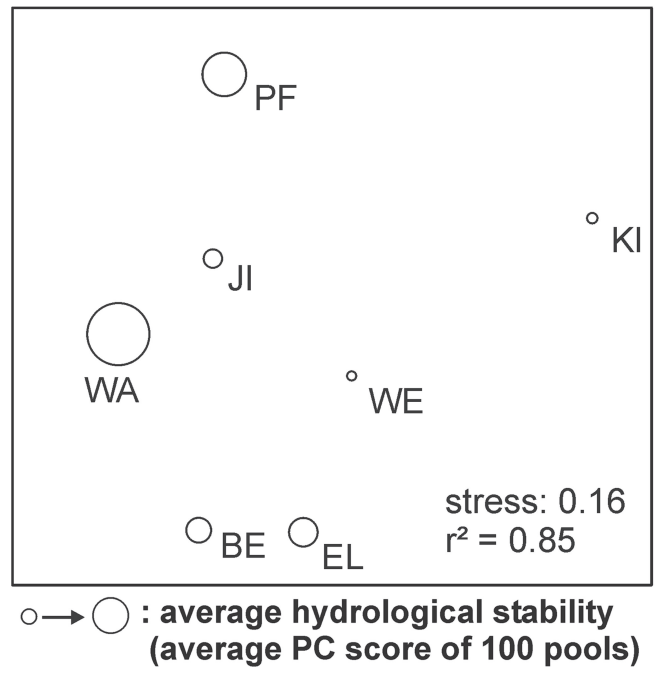

Figure 3. Non metric multidimensional scaling (NMDS) plots illustrating variation in community composition among inselbergs based on the Sørensen dissimilarity index which can be further partitioned in a nestedness fraction (betaSNE) (middle panel) and a turnover fraction (betaSIM) (right panel). NMDS preserves relative distances among sites based on a dissimilarity index but the axes of the ordination are meaningless and unitless. Size of circles is proportional to hydrological stability, which in turn is strongly correlated with latitude (Pearson $r=0.99$ ).

\section{Discussion}

In this study, we set out to test whether environmental stability of patches can explain both local and regional diversity patterns supporting the environmental stability hypothesis as an explanation for the LDG. Specifically, we investigated whether variation in hydrological stability of temporary rock pools could explain variation within as well as among invertebrate metacommunities along a latitudinal gradient.

Our results confirm the presence of a LDG with communities and metacommunities of aquatic macro-invertebrates. Habitats in the northern drier parts of the studied gradient were less diverse than their counterparts in the south of the gradient. It must be noted, however, that, although an LDG could be demonstrated for freshwater macro-invertebrates in this part of Australia, a continent wide analysis will probably result in a different picture. For instance, along a latitudinal gradient in the center of Australia which is characterized by arid conditions in the Nullarbor Plain in the south and moister conditions in the tropical Kimberley region in the north, an opposite pattern could emerge. Additionally, Australian diversity patterns in other taxonomic groups such as birds do not show strong latitudinal gradients but instead reveal strong longitudinal gradients (Pearson and Carroll 1998, Hawkins and Felizola Diniz-Filho 2004) probably due to aridity typically increasing from the coast towards the center of the continent. Therefore the results of this study should not be generalized across the continent. Instead, the strength of this study lies in the simple replicated rock pool model system which allows to explicitly test the mechanisms that generate patterns of aquatic biodiversity along climate gradients which are often - but not necessarily - linked with latitude (López et al. 2013).

At a regional inter-metacommunity scale, an independent assessment of the hydrological stability of individual pools based on pool morphometry, 30 years of climate data and a hydrological model, revealed that latitudinal variation in metacommunity richness can be adequately explained by the average hydrological stability experienced by communities in each metacommunity, supporting variation in hydrological stability as a potential mechanistic explanation for the observed LDG. Desiccation and the time stress associated with short inundations constitute an important environmental filter in temporary pools that may exclude taxa that cannot tolerate frequent drought or fail to mature and reproduce during short inundations (Chase 2007, Vanschoenwinkel et al. 2009). This scenario is consistent with the significantly nested structure of the taxon $X$ inselberg matrix when metacommunities are sorted according to latitude. It has been proposed, however, that in order to reliably assess potential underlying processes to the LDG, it is crucial to show that these processes can lead to similar responses on local scales (Huston 1979, 1999). Such support is provided in this study since variation in hydrological stability of individual pool communities was an excellent predictor of local richness patterns and taxonomic composition. Analogous to the pattern observed at the metacommunity level, communities in less stable habitats were nested in communities in more stable habitats. This suggests that community membership gradually becomes more restricted as conditions become less stable resulting in species loss, as was the case in other systems (Therriault 
and Kolasa 2001, Vanschoenwinkel et al. 2013). It is clear, however, that nestedness does not account for all variation in community composition. Partitioning of community dissimilarity into components of nestedness and turnover revealed that variation in communities and metacommunities also involved turnover of taxa; and that the turnover component contributes more to beta diversity than the nestedness component. Yet, contrary to the latter, turnover showed no clear associations with latitudinal variation in hydrological stability and likely reflects variation in other unmeasured gradients.

In West Australian rock pools, communities seem to be assembled by a core group of disturbance tolerant generalist taxa. These include the fast growing clam shrimp (Paralimnadia), fairy shrimps (Branchinella) and rhabdocoel flatworms (Turbellaria), that are able to bridge dry periods using resistant resting eggs, as well as several actively dispersing insects with drought resistant larval stages such as certain chironomids (Chironomidae) and ceratopogonid midges (Ceratopogonidae). These generalist core taxa are found in metacommunities along the entire latitudinal gradient and are typical both for unstable shallow basins as well as for more stable deeper basins. In turn, a second group of taxa with a higher nested rank are typical for the most stable rock pool habitats and gradually disappear towards the drier north of the gradient. These include several aquatic insects with longer aquatic life phases such as caddisflies (Trichoptera), craneflies (Tipulidae), dragonflies (Hemicordulia) and damselflies (Austrolestes) as well as several crustacean zooplankton genera typical for late succession phases of inundations such as the anomopods Daphnia and Simocephalus. A life history based selective filter linked with hydrological stability of pool habitats, hence, provides an adequate explanation for both local and regional diversity patterns in this system. A similar nested pattern of decreasing richness with reduced stability was observed for oribatid mites in artificial canopy habitats (Lindo et al. 2008). Observed nestedness patterns in this canopy study were interpreted as stochastic dispersal assembly followed by differential survival of disturbance tolerant taxa. A similar non-random pattern of community assembly is plausible for the rock pool communities in this study, even though the spatial and temporal scales are much larger.

This interpretation is reliant on the assumption that dispersal limitation is not an issue and that the spatial scale does not impede colonization. Studies of the genetic structure of poorly dispersed plankton populations in this system show that rock pools on the inselbergs in this study have been colonized for at least several million years and that colonization of all suitable habitats happened in a relatively short window of time (Zofkova and Timms 2009, Pinceel et al. 2013). Additionally, the fact that the taxa which are absent from the less hydrologically stable metacommunities in the north are also absent from shallow unstable habitats in the south is consistent with environmental limitation rather than dispersal limitation. The age of rock pools is also not associated with latitude as geological evidence indicates that inselbergs in this entire region are of a similar age (Twidale and Bourne 1975, 2001).

Consequently, current distributions of taxa at both local and regional scales are probably dominantly driven by species sorting assuming non-limiting dispersal and with establishment success being determined by hydrological stability as a dominant environmental filter (Leibold et al. 2004). At local scales dispersal will be mediated by frequent within cluster dispersal by either active flight - in the case of insects - or passive translocation of propagules by wind and water as measured in previous studies (Vanschoenwinkel et al. 2008). At larger spatial scales, wind might still mediate occasional long distance dispersal of airborne adults or propagules. Yet, particularly water birds such as the Australian maned ducks Chenonetta jubata that occasionally visit rock pools to feed on the quilwort Isoetes (Vanschoenwinkel unpubl.) are likely to be important as efficient vectors transporting the dormant resting eggs of many species from one inselberg to another during their migrations (Green et al. 2008). Although effective long distance dispersal events of passively dispersing freshwater invertebrates among inselbergs has been shown to be very rare - with gene flow occurring roughly once every several 10000 of years (Vanschoenwinkel et al. 2011) - the long geological history of rock pool habitats in this region covering at least several million years makes dispersal limitation over this time scale unlikely. Consequently, when there is enough time for long distance dispersal and colonization, regional processes can be relatively unimportant compared to local processes to explain large scale diversity patterns.

A problem with many studies that try to identify potential contributing processes to the LDG is that many gradients may be correlated to latitude and that systems may not be comparable along the studied gradient. A main strength of the rock pool system is that this is not the case. The model system consists of identical oligotrophic granite pool basins of a similar age. The only clear difference is variation in climate which was shown to be reflected in differences in hydrological stability. A potential contributing role of temperature tolerance alone, independent of hydrology, however cannot be excluded based on the data at hand. Additionally, it is also possible that hydrology might have indirect effects on diversity via other unmeasured variables such as nutrients, but this is speculative. Ultimately, our results show that the same environmental filter - in this case hydrological stability - can explain both small and large scale diversity patterns and highlights the potential role environmental stability can play at these different scales. This research also illustrates the merits of spatial hierarchical model systems such as the studied pool clusters to investigate diversity patterns over different spatial scales in a natural environment, which has thus far not been fully exploited (but see Sfenthourakis et al. 2004, Declerck et al. 2011). Such knowledge is highly relevant in the context of the emerging synthesis between ecology and biogeography (Jenkins and Ricklefs 2011), which aims to identify common and unique processes that can generate diversity at different spatial and temporal scales.

Acknowledgements - LB and BV contributed equally to this manuscript. We would like to thank Mark Urban, Steven Declerck, Karl Cottenie, Tom Pinceel and Falko Buschke for constructive comments on earlier versions of the manuscript. We are indebted to Russell Mauritz and family for providing accommodation during our stay and ready access to Wave Rock and to Nooike Alyn and Bruce Riddoch for help in the field. This research was financially supported by projects G.0118.03 of the FWO (Fund for scientific Research Flanders). A travel scholarship was provided by the King Leopold III Fund for Nature Exploration and Conservation. 


\section{References}

Almeida-Neto, M. et al. 2008. A consistent metric for nestedness analysis in ecological systems: reconciling concept and measurement. - Oikos 117: 1227-1239.

Altermatt, F. et al. 2009. Desiccation of rock pool habitats and its influence on population persistence in a Daphnia metacommunity. - PloS ONE 4:e4703.

Baselga, A. 2010. Partitioning the turnover and nestedness components of beta diversity. - Global Ecol. Biogeogr. 19: 134-143.

Baselga, A. and Orme, C. D. L. 2012. betapart: an R package for the study of beta diversity. - Meth. Ecol. Evol. 3: 808-812.

Bayly, I. A. E. 2011. Australia's granite wonderlands: rock of ages' intriguing landscapes and life. - BAS Publishing.

Borcard, D. et al. 2011. Numerical ecology with R. - Springer.

Brualdi, R. A. and Sanderson, J. G. 1999. Nested species subsets, gaps, and discrepancy. - Oecologia 119: 256-264.

Cardillo, M. et al. 2005. Testing for latitudinal bias in diversification rates: an example using New World birds. - Ecology 86: 2278-2287.

Chase, J. M. 2007. Drought mediates the importance of stochastic community assembly. - Proc. Natl Acad. Sci. USA 104: $17430-17434$.

Declerck, S. A. J. et al. 2011. Scale dependency of processes structuring metacommunities of cladocerans in temporary pools of High-Andes wetlands. - Ecography 34: 296-305.

Fox, J. W. 2013. The intermediate disturbance hypothesis should be abandoned. - Trends Ecol. Evol. 28: 86-92.

Green, A. J. et al. 2008. The potential role of waterbirds in dispersing invertebrates and plants in arid Australia. - Freshwater Biol. 53: 380-392.

Hawkins, B. A. and Felizola Diniz-Filho, J. A. 2004. 'Latitude' and geographic patterns in species richness. - Ecography 27 : 268-272.

Hillebrand, H. 2004. On the generality of the latitudinal diversity gradient. - Am. Nat. 163: 192-211.

Hulsmans, A. et al. 2008. Quantifying the hydroregime of a temporary pool habitat: a modelling approach for ephemeral rock pools in SE Botswana. - Ecosystems 11: 89-100.

Huston, M. 1979. A general hypothesis of species diversity. - Am. Nat. 113: 81-101.

Huston, M. A. 1999. Local processes and regional patterns: appropriate scales for understanding variation in the diversity of plants and animals. - Oikos 86: 393-401.

Jenkins, D. G. and Ricklefs, R. E. 2011. Biogeography and ecology: two views of one world. - Phil. Trans. R. Soc. B. 366: 2331-2335.

Jocqué, M. et al. 2007. A contribution on the biodiversity and conservation of the freswhater fauna of rocky outcrops in the central wheatbelt of Western Australia. - Proc. R. Soc. W. Aust. 90: $137-147$.

Jocqué, M. et al. 2010. Climatic control of dispersal-ecological specialization tradeoffs: a metacommunity process at the heart of the latitudinal diversity gradient? - Global Ecol. Biogeogr. 19: 244-252.

Leibold, M. A. et al. 2004. The metacommunity concept: a framework for multi-scale community ecology. - Ecol. Lett. 7: 601-613.

Legendre, P. and Gallagher, E. D. 2001. Ecologically meaningful transformations for ordination of species data. - Oecologia 129: 271-280.

Lindo, Z. et al. 2008. Nested patterns of community assembly in the colonisation of artificial canopy habitats by orbatid mites. Oikos 177: 1856-1864.

López, R. P. et al. 2013. Co-occurrence patterns along a regional aridity gradient of the subtropical Andes do not support stress gradient hypotheses. - PLoS ONE 8: e58518.

Supplementary material (available as Appendix oik.01710 at $<$ www.oikosjournal.org/readers/appendix $>$ ). Appendix 1 .
Mackey, R. L. and Currie, D. J. 2001. The diversity-disturbance relationship: is it generally strong and peaked? - Ecology 82: 3479-3492.

Mittelbach, G. G. et al. 2007. Evolution and the latitudinal diversity gradient: speciation, extinction and biogeography. - Ecol. Lett. 10: 315-331.

Pearson, D. L. and Carroll, S. S. 1998. Global patterns of species richness: spatial models for conservation planning using bioindicator and precipitation data. - Conserv. Biol. 12: 809-821.

Pinceel, T. et al. 2013. Environmental change as a driver of diversification in temporary aquatic habitats - does the genetic structure of extant fairy shrimp populations reflect historic aridification? - Freshwater Biol. 58: 1556-1572.

Rodríguez-Gironés, M. A. and Santamaría, L. 2006. A new algorithm to calculate the nestedness temperature of presenceabsence matrices. - J. Biogeogr. 33: 924-935.

Schneider, D. W. and Frost, T. M. 1996. Habitat duration and community structure in temporary ponds. - J. N. Am. Benthol. Soc. 15: 64-86.

Sfenthourakis, S. et al. 2004. From sampling stations to archipelagos: investigating aspects of the assemblage of insular biota. - Global Ecol. Biogeogr. 13: 23-35.

Therriault, T. W. and Kolasa, J. 2001. Desiccation frequency reduces species diversity and predictability of community structure in coastal rock pools. - Isr. J. Zool. 47: 477-489.

Timms, B. V. 2012. Seasonal study of aquatic invertebrates in five sets of latitudinally separated gnammas in southern Western Australia. - J. R. Soc. W. Austr. 95: 13-28.

Tuytens, K. et al. 2014. Predictions of climate change infer increased environmental harshness and altered connectivity in a cluster of temporary pools. - Freshwater Biol. 59: 955-968.

Twidale, C. and Bourne, J. 1975. Episodic exposure of inselbergs. Geol. Soc. Am. Bull. 86: 1473-1481.

Twidale, C. R. and Bourne, J. A. 2001. A field guide to Hyden rock, Western Australia. - Wave Rock Management, P/L Hyden, Western Australia, Australia.

Urban, M. C. 2004. Disturbance heterogeneity determines freshwater metacommunity structure. - Ecology 85: 2971-2978.

Ulrich, W. and Gotelli, N. J. 2007. Null model analysis of species nestedness patterns. - Ecology 88: 1824-1831.

Vanschoenwinkel, B. et al. 2007. The role of metacommunity processes in shaping invertebrate rock pool communities along a dispersal gradient. - Oikos 116: 1255-1266.

Vanschoenwinkel, B. et al. 2008. Relative importance of different dispersal vectors for small aquatic invertebrates in a rock pool metacommunity. - Ecography 31: 567-577.

Vanschoenwinkel, B. et al. 2009. Community structure in temporary freshwater pools: disentangling effects of habitat size and hydroregime. - Freshwater Biol. 54: 1487-1500.

Vanschoenwinkel, B. et al. 2011. Long distance dispersal of zooplankton endemic to isolated mountaintops - an example of an ecological process operating on an evolutionary time scale. PloS ONE 6:e26730.

Vanschoenwinkel, B. et al. 2013. Disturbance regime alters the impact of dispersal on alpha and beta diversity in a natural metacommunity. - Ecology 94: 2547-2557.

Wallace, A. R. 1878. Tropical nature and other essays. - Macmillan.

Wellborn, G. A. et al. 1996. Mechanisms creating community structure across a freshwater habitat gradient. - Annu. Rev. Ecol. Syst. 27: 337-363.

Wiggins, G. B. et al. 1980. Evolutionary and ecological strategies of animals in annual temporary pools. - Arch. Hydrobiol. 58: 97-206.

Withers, P. C. 2000. Overview of granite outcrops in Western Australia. - J. R. Soc. W. Aust. 83: 103-108.

Zofkova, M. and Timms, B. V. 2009. A conflict of morphological and genetic patterns in the Australian anostracan Branchinella longirostris. - Hydrobiologia 635: 67-80. 